\title{
BIBLIOGRAPHIGAL ABBREVIATIONS
}

\section{Proceedings of Meetings on Mycenaean Studies.}

Etudes Mycéniennes = Etudes Mycéniennes. Actes du Colloque International sur les Textes Mycéniens (Gif-surIvette, 3-7 avril 1956), ed. M. Lejeune, Paris 1956.

Atti Pavia

$=$ Atti del 2.0 Colloquio Internazionale di Studi Minoico-Micenei (Pavia, 1-5 IX 1958) = Athenaeum 46. 1958, pp. 295-440.

Wingspread Colloquium $=$ Mycenaean Studies. Proceedings of the Third International Colloquium for Mycenaean Studies held at «Wingspread», 4-8 September 1961, ed. E. L. Bennett, jr., Madison 1964.

Cambridge Colloquium $=$ Proceedings of the Cambridge Colloquium on Mycenaean Studies, edd. L. R. Palmer and J. Chadwick, Cambridge 1966.

Atti Roma

$=$ Atti e memorie del Io Congresso Internazionale di Micenologia, Roma 27 settembre - 3 ottobre 1967, 3 vols., Roma 1968.

Studia Mycenaea Brno = Studia Mycenaea. Proceedings of the Mycenaean Symposium, Brno April 1966, ed. A. Bartoněk, Brno 1968.

II. Texts.

ICS

=- Les inscriptions chypriotes syllabiques, ed. O. Masson, Paris 1961.

IP

$=$ Inscriptiones Pyliae, edd. C. Gallavotti and A. Sacconi, Roma 1961.

$K T^{2}$

$=$ The Knossos Tablets, A Transliteration by E. L. Bennett, jr., J. Chadwick and M. Ventris, London 1959. 
$=$ The Knossos Tablets $^{3}$, A Transliteration by J. Chadwick and J. T. Killen, London 1964.

$K T^{4}$

$=$ The Knossos Tablets IV, A Transliteration by J. Ghadwick, J. T. Killen and J.-P.

$M T I I$ Olivier, London 1971.

MT III

$=$ The Mycenae Tablets II, ed. E. L. Bennett, jr., TAPhS 48, 1958.

$=$ The Mycenae Tablets III, ed. J. Chadwick, TAPhS 52, 1962.

MT IV = The Mycenae Tablets IV, A Revised Transliteration by J.-P. Olivier, Leiden 1969.

Olive Oil Tablets = The Olive Oil Tablets of Pylos, Text of the Inscriptions Found, 1955, ed. E. L. Bennett, jr., Salamanca 1958.

PT II

$=$ The Pylos Tablets II: Texts of the Inscriptions Found, 1939-1954, ed. E. L. Bennett, jr., Princeton 1955.

$P T \mathcal{T}$

$=$ The Pylos Tablets Transliterated, edd. E. L. Bennett, jr., and J.-P. Olivier [not yet published].

$S M I I$

$=$ Scripta Minoa II, by A. J. Evans, ed. J. L. Myres, Oxford 1952.

Vases

$=\mathrm{J}$. Raison, Les vases à inscriptions peintes de l'âge mycénien et leur contexte archéologique, Rome 1967.

III. OTHER WORKs.

Avviamento

Demiourgos

Desservants

Development
$=$ M. Doria, Avviamento allo studio del miceneo: struttura, problemi e testi, Roma 1965.

$=\mathrm{F}$. Bader, Les composés grecs du type de demiourgos, Paris 1965.

$=\mathrm{J} .-\mathrm{P}$. Olivier, $A$ propos d'une «liste» de desservants de sanctuaire dans les documents en linéaire B de Pylos, Bruxelles 1960.

$=$ A. Bartoněk, Development of the LongVowel System in Ancient Greek Dialects, Brno 1966. 
Dictionnaire

Documents

Dornseiff-Hansen

Etudes

Europa

Evidence

Formation

GEW

Grammar

Griech. Gramm.

$H P \mathcal{N}$

IEW

Index inverse

Interpretation

Lex.

Lexicon
$=\mathrm{P}$. Chantraine, Dictionnaire étymologique de la langue grecque. Histoire des mots. Paris 1968-.

$=\mathrm{M}$. Ventris-J. Chadwick, Documerts in Mycenaean Greek, Cambridge 1956.

$=$ F. Dornseiff-B. Hansen, Rückläufïges Wörterbuch der griechischen Eigennamen, Berlin 1957.

= C. J. Ruijgh, Etudes sur la grammaire et le vocabulaire du grec mycénien, Amsterdam 1967.

$=$ Europa. Studien zur Geschichte und Epigraphik der frühen Ägäis. Festschrift für $E$. Grumach, ed. W. C. Brice, Berlin 1967.

$=\mathrm{M}$. Ventris-J. Chadwick, «Evidence for Greek Dialect in the Mycenaean Archives», FHS 73, 1953, pp. 84-103.

$=\mathrm{P}$. Chantraine, La formation des noms en grec ancien, Paris 1933.

$=\mathrm{Hj}$. Frisk, Griechisches etymologisches Wörterbuch, Heidelberg 1954-.

$=$ E. Vilborg, A Tentative Grammar of Mycenaean Greek, Göteborg 1960.

$=$ E. Schwyzer, Griechische Grammatik, München 1934-1953.

= F. Bechtel, Die historischen Personennamen des Griechischen bis zur Kaiserzeit, Halle 1917.

$=\mathrm{J}$. Pokorny, Indogermanisches etymologisches Wörterbuch, Bern 1949-1959.

$=$ M. Lejeune, Index inverse du grec mycénien, Paris 1964.

$=$ L. R. Palmer, The Interpretation of Mycenaean Greek Texts, Oxford 1963.

= Vl. Georgiev, Lexique des inscriptions crétomycéniennes, Sofia 1955.

= A. Morpurgo, Mycenaeae Graecitatis Lexicon, Roma 1963. 


\begin{tabular}{|c|c|}
\hline$L f g r E$ & $\begin{array}{l}=\text { B. Snell, Lexikon des frühgriechischen Epos, } \\
\text { Göttingen 1955-. }\end{array}$ \\
\hline$L S \mathcal{J}$ & $\begin{aligned}= & \text { H. G. Liddell, R. Scott and H. St. Jones, } \\
& \text { A Greek-English Lexicon }{ }^{9} \text {, Oxford } 1940 . \\
& \text { (With a Supplement, 1968). }\end{aligned}$ \\
\hline Mémoires & $\begin{aligned}= & \text { M. Lejeune, Mémoires de philologie mycé- } \\
& \text { nienne I, Paris } 1958 .\end{aligned}$ \\
\hline Mentions & $\begin{aligned}= & M . \text { Gérard-Rousseau, Les mentions reli- } \\
& \text { gieuses dans les tablettes mycéniennes, Rome } \\
& 1968 .\end{aligned}$ \\
\hline Minoica & $\begin{aligned}= & \text { Minoica. Festschrift für } \mathcal{F} . \text { Sundwall, Berlin } \\
& 1958 .\end{aligned}$ \\
\hline$M L B$ Index & $\begin{array}{l}=\text { E. L. Bennett, jr., } A \text { Minoan Linear } B \\
\text { Index, New Haven } 1953 \text {. }\end{array}$ \\
\hline Pape-Benseler & $\begin{aligned}= & \text { W. Pape-G. E. Benseler, Wörterbuch der } \\
& \text { griechischen Eigennamen }{ }^{3} \text {, Braunschweig } \\
& 1884 .\end{aligned}$ \\
\hline Personennamen & $\begin{aligned}= & \text { O. Landau, Mykenisch-griechische Personen- } \\
& \text { namen, Göteborg } 1958 .\end{aligned}$ \\
\hline Rev. Index & $\begin{array}{l}=\text { G. D. Buck-W. Petersen, A Reverse Index } \\
\text { of Greek Nouns and Adjectives, Chicago } \\
\text { 1944. }\end{array}$ \\
\hline Scribes & $\begin{aligned}= & \text { J.-P. Olivier, Les scribes de Cnossos, Rome } \\
& 1957 .\end{aligned}$ \\
\hline Studies & $\begin{aligned}= & \text { L. Baumbach, Studies in Mycenaean Inscrip- } \\
& \text { tions and Dialect, 1953-1964, Rome 1968. }\end{aligned}$ \\
\hline Suppl. I & $\begin{aligned}= & \text { Vl. Georgiev, Supplément au lexique des } \\
& \text { inscriptions créto-mycéniennes, Sofia } 1955 / 6 .\end{aligned}$ \\
\hline Suppl. II & $\begin{array}{l}=\text { Vl. Georgiev, Second supplément au lexique } \\
\text { des inscriptions créto-mycéniennes, Sofia } \\
\text { 1955/6. }\end{array}$ \\
\hline Traité $^{2}$ & $\begin{aligned}= & \text { M. Lejeune, Traité de phonétique grecque }{ }^{2} \text {, } \\
& \text { Paris } 1955 .\end{aligned}$ \\
\hline Vocabulary & $\begin{aligned} &= \text { J. Chadwick - L. Baumbach, «Myce- } \\
& \text { naean Greek Vocabulary», Glotta 41, } \\
& \text { 1963, pp. 157-2.71. }\end{aligned}$ \\
\hline Vývoj & $\begin{aligned}= & \text { A. Bartoněk, Vývoj konsonantického systému } \\
& \text { v reckých dialektech, Praha } 1961 .\end{aligned}$ \\
\hline
\end{tabular}

